\title{
Ursodeoxycholic Acid in Treatment of Non-cholestatic Liver Diseases: A Systematic Review
}

\author{
Jillian Reardon ${ }^{1}$, Trana Hussaini ${ }^{2,3}$, Majid Alsahafi ${ }^{2}$, Vladimir Marquez Azalgara ${ }^{2}$, \\ Siegfried R. Erb ${ }^{2}$, Nilufar Partovi ${ }^{1,3}$ and Eric M. Yoshida*2

\begin{abstract}
${ }^{1}$ Faculty of Pharmaceutical Sciences, The University of British Columbia, Vancouver, British Columbia, Canada; ${ }^{2}$ Division of Sciences Clinical Service Unit, Vancouver General Hospital, Vancouver, British Columbia, Canada
\end{abstract} \\ Gastroenterology, The University of British Columbia, Diamond Health, Vancouver, British Columbia, Canada; ${ }^{3}$ Pharmaceutical
}

\begin{abstract}
Aims: To systematically evaluate the literature for evidence to support the use of bile acids in non-cholestatic liver conditions. Methods: Searches were conducted on the databases of Medline (1948-March 31, 2015), Embase (1980-March 31, 2015) and the Cochrane Central Register of Controlled Trials, and on Google and Google Scholar to identify articles describing ursodeoxycholic acid (UDCA) and its derivatives for noncholestatic hepatic indications. Combinations of the following search terms were used: ursodeoxycholic acid, ursodiol, bile acids and/or salts, non alcoholic fatty liver, non alcoholic steatohepatitis, fatty liver, alcoholic hepatitis, alcohol, liver disease, autoimmune, autoimmune hepatitis, liver transplant, liver graft, transplant rejection, graft rejection, ischemic reperfusion injury, reperfusion injury, hepatitis $B$, hepatitis $C$, viral hepatitis, chronic hepatitis, acute hepatitis, transaminases, alanine transaminase, liver enzymes, aspartate aminotransferase, gamma-glutamyl transferase, gammaglutamyl transpeptidase, bilirubin, alkaline phosphatase. No search limits were applied. Additionally, references of the included studies were reviewed to identify additional articles. Results: The literature search yielded articles meeting inclusion criteria for the following indications: non-alcoholic fatty liver disease $(n=5)$; alcoholic liver disease $(n=2)$; autoimmune hepatitis $(n=6)$, liver transplant $(n=2)$ and viral hepatitis $(n=9)$. Bile acid use was associated with improved normalization of liver biochemistry in non-alcoholic fatty liver disease, autoimmune hepatitis and hepatitis B and C infections. In contrast, liver biochemistry normalization was inconsistent in alcoholic liver disease and liver transplantation. The
\end{abstract}

Keywords: Non-alcoholic fatty liver disease; Alcoholic liver disease; Autoimmune hepatitis; Liver transplantation; Viral hepatitis.

Abbreviations: UDCA, ursodeoxycholic acid; $\mathrm{HCC}$, hepatocellular carcinoma; $\mathrm{PBC}$, primary biliary cirrhosis; PSC, primary sclerosing cholangitis; NAFLD, nonalcoholic fatty liver disease; ALD, alcoholic liver disease; AIH, autoimmune hepatitis; HBV, hepatitis B virus; $\mathrm{HCV}$, hepatitis $\mathrm{C}$ virus; SR, systematic review; MA meta-analysis; RCT, randomized controlled trial; $A L T$, alanine transaminase; AST, aspartate aminotransferase; GGT, gamma-glutamyl transferase; NAS, nonalcoholic fatty liver disease activity score; NASH, non-alcoholic steatohepatitis; ALP, alkaline phosphatase; Ig, immunoglobulin; ANA, antinuclear antibody; HLA human leukocyte antigen; TUDCA, tauro-ursodeoxycholic acid; ACR, acute cellular rejection; MHC, major histocompatibility complex; IRI, ischemic reperfusion injury; ASMA, anti-smooth muscle antibodies.

Received: 26 May 2016; Revised: 30 June 2016; Accepted: 12 July 2016

DOI: $10.14218 / \mathrm{JCTH} .2016 .00023$

*Correspondence to: Eric M. Yoshida, Division of Gastroenterology, Vancouver General Hospital, 5153-2775 Laurel Street, Vancouver, BC V5Z 1M9, Canada. Tel: +1-604-875-5371, Fax: +1-604-875-5447, E-mail: eric.yoshida@vch.ca majority of studies reviewed showed that normalization of liver biochemistry did not correlate to improvement in histologic disease. In the prospective trials reviewed, adverse effects associated with the bile acids were limited to minor gastrointestinal complaints (most often, diarrhea) and did not occur at increased frequency as compared to controls. As administration of bile acids was often limited to durations of 12 months or less, long-term side effects for non-cholestatic indications cannot be excluded. Conclusions: Based on the available literature, bile acids cannot be widely recommended for noncholestatic liver diseases at present.

(C) 2016 The Second Affiliated Hospital of Chongqing Medical University. Published by XIA \& HE Publishing Inc. All rights reserved.

\section{Introduction}

Ursodeoxycholic acid (UDCA) is a non-toxic, hydrophilic bile acid indicated for treatment of gallstones and primary biliary cirrhosis (PBC). ${ }^{1}$ Endogenous bile acids are hepatically synthesized and regulate cholesterol homeostasis and solubilization of dietary lipids. Chenodeoxycholic and deoxycholic acid-the two major human bile acids-are hydrophobic, and when in excess contribute to direct biliary toxicity through their detergent effects on lipid membranes. ${ }^{2}$ UDCA is naturally occurring in humans, comprising only $1-3 \%$ of the total bile acid pool. When used in treatment of PBC, doses of $13-15 \mathrm{mg} / \mathrm{kg} / \mathrm{day}$ increase the concentration to $40-60 \%$, making UDCA the predominant bile acid. Increasing bile pool hydrophilicity via UDCA serves to improve cholestasis and minimize toxicity. ${ }^{3}$ Additionally, UDCA is postulated to possess other pharmacologic mechanisms, including stimulation of hepatocellular and biliary ductular secretions, and to exert anti-inflammatory effects, making it attractive as treatment for a multitude of liver diseases. Moreover, studies have demonstrated that UDCA has efficacy in reducing histologic progression of PBC, as well as the need for liver transplantation and survival. ${ }^{4}$ While UDCA may be prescribed for other cholestatic conditions (i.e. primary sclerosing cholangitis (PSC), cholestasis of pregnancy and cystic fibrosis, and graft versus host disease), the supporting data reported to date is minimal and limited predominantly to surrogate biochemical markers. ${ }^{5,6}$ Furthermore, although UDCA is anecdotally used for non-cholestatic liver diseases, wherein liver biochemistry is frequently abnormal, the practice guidelines do not endorse its use. 
The objective of this review was to systemically evaluate the literature to ascertain evidence for UDCA in the following non-cholestatic liver diseases: non-alcoholic fatty liver disease (NAFLD), alcoholic liver disease (ALD), autoimmune hepatitis $(\mathrm{AIH})$, liver transplantation, and acute and chronic infections with hepatitis B (HBV) and/or C (HCV).

\section{Methods}

\section{Literature search}

Searches of the Medline (1948-March 31, 2015) and Embase (1980-March 31, 2015) databases, the Cochrane Central Register of Controlled Trials, Google and Google Scholar were conducted to identify articles describing UDCA and derivatives for non-cholestatic hepatic indications. Separate searches were conducted for each condition using the following combinations of both free-text and MeSH terms: ursodeoxycholic acid and/or ursodiol and/or bile acids and/or salts 'and': 1 . non alcoholic fatty liver and/or non alcoholic steatohepatitis and/or fatty liver; 2. alcoholic hepatitis and/or alcohol and liver disease; 3. autoimmune and/or autoimmune hepatitis; 4. liver transplant and/or liver graft and/or transplant rejection and/or graft rejection; 5 . ischemic reperfusion injury and/or reperfusion injury; 6 . hepatitis $B$ and/or hepatitis $C$ and/or viral hepatitis and/or chronic hepatitis and/or acute hepatitis; 7. ursodeoxycholic acid and/or ursodiol and/or bile acids and/or salts 'and' transaminases and/or alanine transaminase and/or liver enzymes and/or aspartate aminotransferase and/ or gamma-glutamyl transferase and/or gamma-glutamyl transpeptidase and/or bilirubin and/or alkaline phosphatase. No search limits were applied. The references lists of the retrieved studies were also reviewed to identify any additional articles that might meet our inclusion criteria.

\section{Study selection}

Randomized controlled trials (RCTs) and observational studies (i.e. cohort, case-control and case series) evaluating UDCA and derivatives in adults to treat the following non-cholestatic liver conditions were selected for inclusion in the study: NAFLD, ALD, AIH, liver transplant complication prophylaxis or treatment and acute or chronic HBV and HCV. Studies with the following characteristics were excluded: non-human, non-English language, publication only in abstract form, pediatric patients exclusively and bile acid use in purely cholestatic liver conditions such as PBC or PSC. No limitations were placed on trial quality.

\section{Data extraction and evaluation}

The following data were extracted from each included study: design, participant number, inclusion and exclusion criteria, baseline characteristics, drug dosing regimens, study outcomes and conclusions.

\section{Results/Discussion}

The search yielded 24 articles meeting inclusion criteria for the following indications: NAFLD $(n=5)$; ALD $(n=2)$; AIH $(n=6)$, liver transplant $(n=2)$ and viral hepatitis $(n=9)$. Tables 1-5 summarize the details of the individual trials.

\section{NAFLD}

Five publications comprising 1447 patients to examine the use of UDCA for patients with NAFLD were included, represented by 1 systematic review (SR) and meta-analysis (MA) of 12 RCTs, 2 RCTs not included in this MA, 1 observational trial and 1 non-RCT. ${ }^{7-11}$ UDCA doses ranged from $13-28 \mathrm{mg} /$ $\mathrm{kg} /$ day for durations of 3 months to over 5 years.

\section{Biochemistry}

All studies evaluated UDCA impact on liver biochemistry. In the SR and 2 RCTs, compared to placebo or no therapy, UDCA was associated with greater improvement in one or more of: alanine transaminase (ALT), aspartate aminotransferase (AST), gamma-glutamyl transferase (GGT). ${ }^{7-9,11}$ Combination therapy of UDCA with vitamin $\mathrm{E} \pm$ vitamin $\mathrm{C}$, polyene phosphatidylcholine, silymarin, glycyrrhizin or tiopronin was associated with greater biochemistry normalization than the monotherapy. ${ }^{7}$ Most data for combination therapy employed vitamin $E{ }^{8,10}$ Results were conflicting if high- versus low-dose UDCA conferred a greater benefit, with one study reporting a significant reduction in serum glucose, glycosylated hemoglobin and serum insulin concentrations at doses of $28-35 \mathrm{mg} /$ $\mathrm{kg} /$ day. $^{7}$ Improved glycemic control with UDCA at lower doses was reported by two additional trials. ${ }^{9,11}$

\section{Histology}

Four of the studies included in the SR and an additional RCT reported improvement in liver steatosis and fibrosis with UDCA therapy. ${ }^{7,11}$ Additionally, Pietu et $\mathrm{al}^{8}$ described 7 patients from their initial cohort with 5-year biopsies demonstrating an average improvement of -1 point on the 8-point NAFLD activity score (NAS) scale.

\section{Summary}

NAFLD is the most common liver disease in Western countries and encompasses a spectrum of liver pathology, ranging from steatosis to non-alcoholic steatohepatitis (NASH). A small percentage of NASH patients progress to liver cirrhosis and subsequent hepatocellular carcinoma (HCC). Risk factors for NAFLD include visceral obesity, insulin resistance, hypertension and hyperlipidemia (i.e. metabolic syndrome). There are currently no liver-specific pharmacological therapies for NAFLD and management focuses on diet and lifestyle modification and pharmacologic management of the diseases comprising the metabolic syndrome. ${ }^{12}$ Underlying pathological mechanisms causing NAFLD are not fully understood. Abnormal lipid metabolism and dysregulation of proinflammatory species likely contribute to disease progression. As a result, it is plausible that exogenous administration of a non-toxic bile acid, such as UDCA, may be cytoprotective. Animal models of UDCA in NAFLD have demonstrated antiapoptotic and mitochondrial protective effects as well as reductions in pro-inflammatory cytokines, such as TNF-alpha. ${ }^{13-15}$ A less obvious role for UDCA in NAFLD is insulin sensitization; although the mechanism is unknown, improved glycemic control has been demonstrated in animal models and trials that are included in this review. ${ }^{14}$

While ALT and AST are typically elevated 3-5 times the upper normal limit in NAFLD, clinically significant histologic injury can occur with normal transaminases. ${ }^{16}$ Most patients 


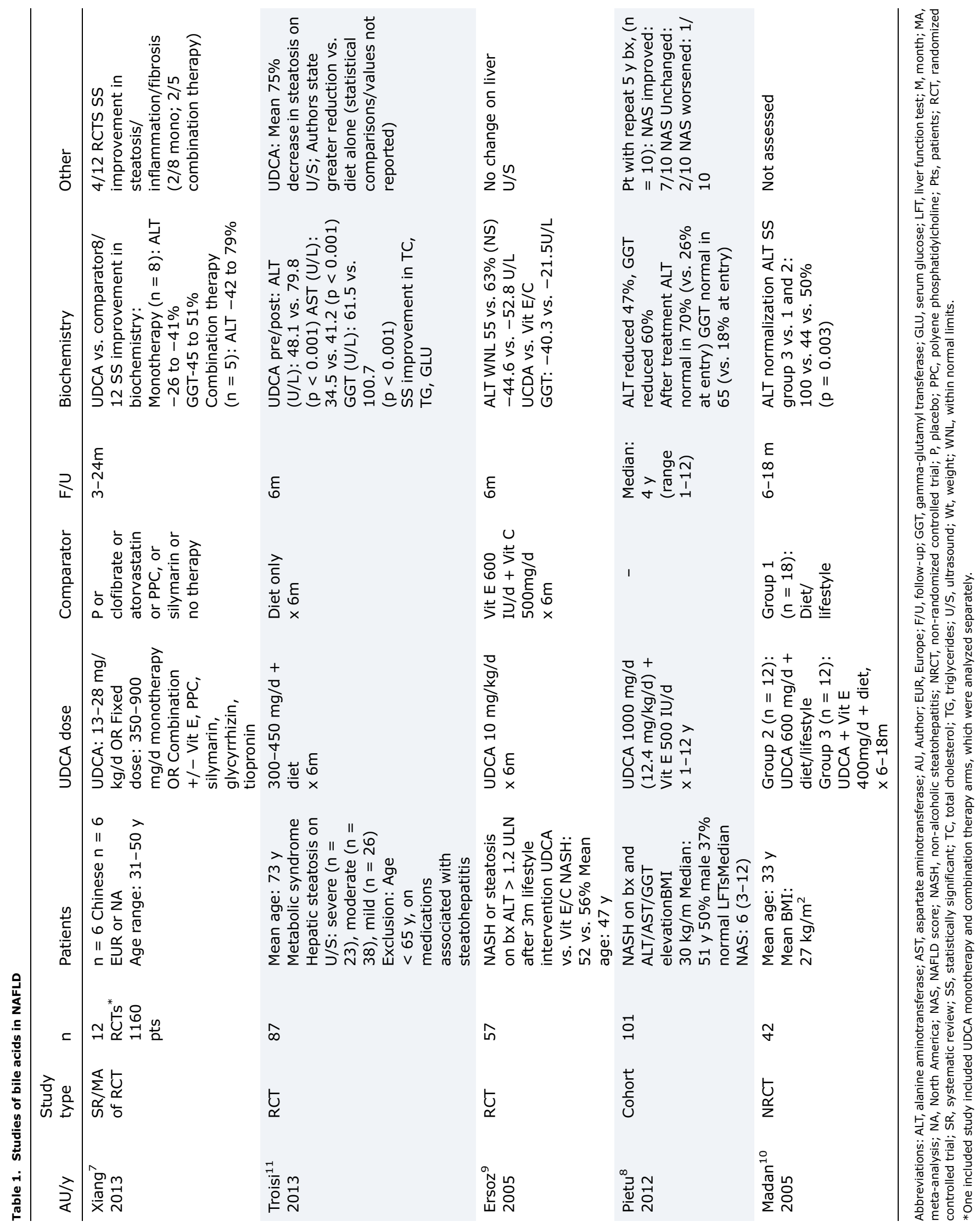




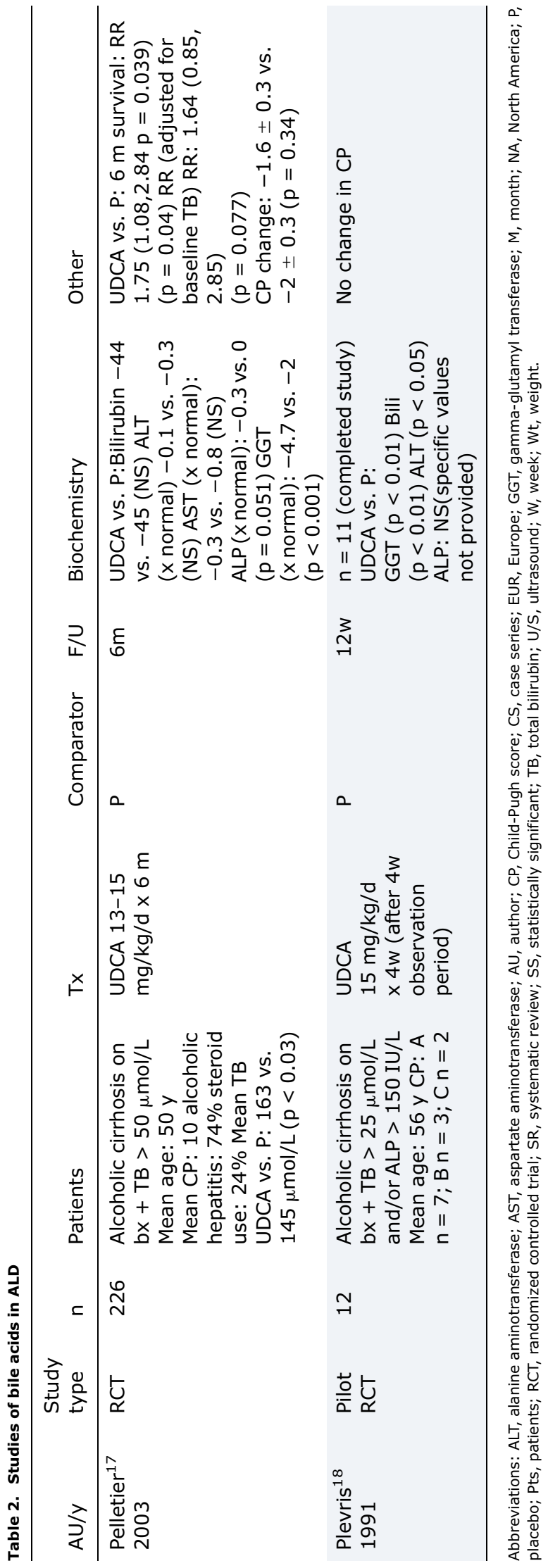

in the trials included herein were reported to have baseline transaminase elevations. In addition, the majority of studies demonstrated a significant reduction in liver transaminases with UDCA compared to controls; however, this was not consistently associated with histologic improvement. Based on the available data, histologic impact of aggressive normalization of transaminases with UDCA is unknown.

The included studies were limited by heterogeneity, as evidenced by the inability of Xiang et al, ${ }^{7}$ authors of the large systematic review, to meta-analyze their data. In that SR, the average study quality, as rated by investigators on a 5-point scale, was 2.69 , with many obvious methodological flaws, including lack of blinding in several trials. In all the included studies, diagnostic criteria for NAFLD were variable, with a wide spectrum of disease severity and inconsistent diagnostic biopsy use. Additionally, lifestyle interventions were inconsistent or not controlled. This creates significant potential for confounding and may obscure the true effect of UDCA. Similarly, studies reporting histology improvements frequently combined UDCA with vitamin supplements, thereby precluding accurate assessment of the monotherapy.

Currently, there is insufficient evidence to recommend widespread use of UDCA in patients with NAFLD. UDCA administration was not associated with harm over prolonged periods. Given the minimal risk, a trial of UDCA in patients with NAFLD and persistently elevated transaminases and poor glycemic control may be justified. UDCA doses should be $13-15 \mathrm{mg} / \mathrm{kg} / \mathrm{day}$ and discontinued if biochemical normalization is not achieved within 3-6 months.

\section{ALD}

Two RCTs comprising 238 patients studied UDCA in ALD. ${ }^{17,18}$ Doses ranged from $13-15 \mathrm{mg} / \mathrm{kg} /$ day, with durations of 4 weeks to 6 months. All patients had biopsy-confirmed liver cirrhosis and the majority continued to consume alcohol throughout follow-up.

\section{Biochemistry}

ALT and bilirubin were significantly reduced with UDCA in one trial. Moreover, the reduction was proportional to underlying liver disease severity. Bilirubin returned to pre-treatment levels upon UDCA cessation. ${ }^{18}$ GGT was significantly reduced compared to baseline in both trials. ${ }^{17,18}$

\section{Histology}

Histologic data, beyond the initial liver biopsy to confirm diagnosis, was not collected in either trial.

\section{Other clinical outcomes}

Pelletier et $\mathrm{al}^{17}$ found no difference in 6-month survival between UDCA and placebo. Plevris et $\mathrm{al}^{18}$ found no difference in Child-Pugh scores pre- and post-UDCA administration.

\section{Summary}

Excessive alcohol consumption is responsible for development of alcoholic fatty liver disease, alcoholic hepatitis and cirrhosis, all of which are termed ALD. ALD is diagnosed based on history of alcohol excess and evidence of liver disease. Often, transaminases will be elevated with a classic pattern of 


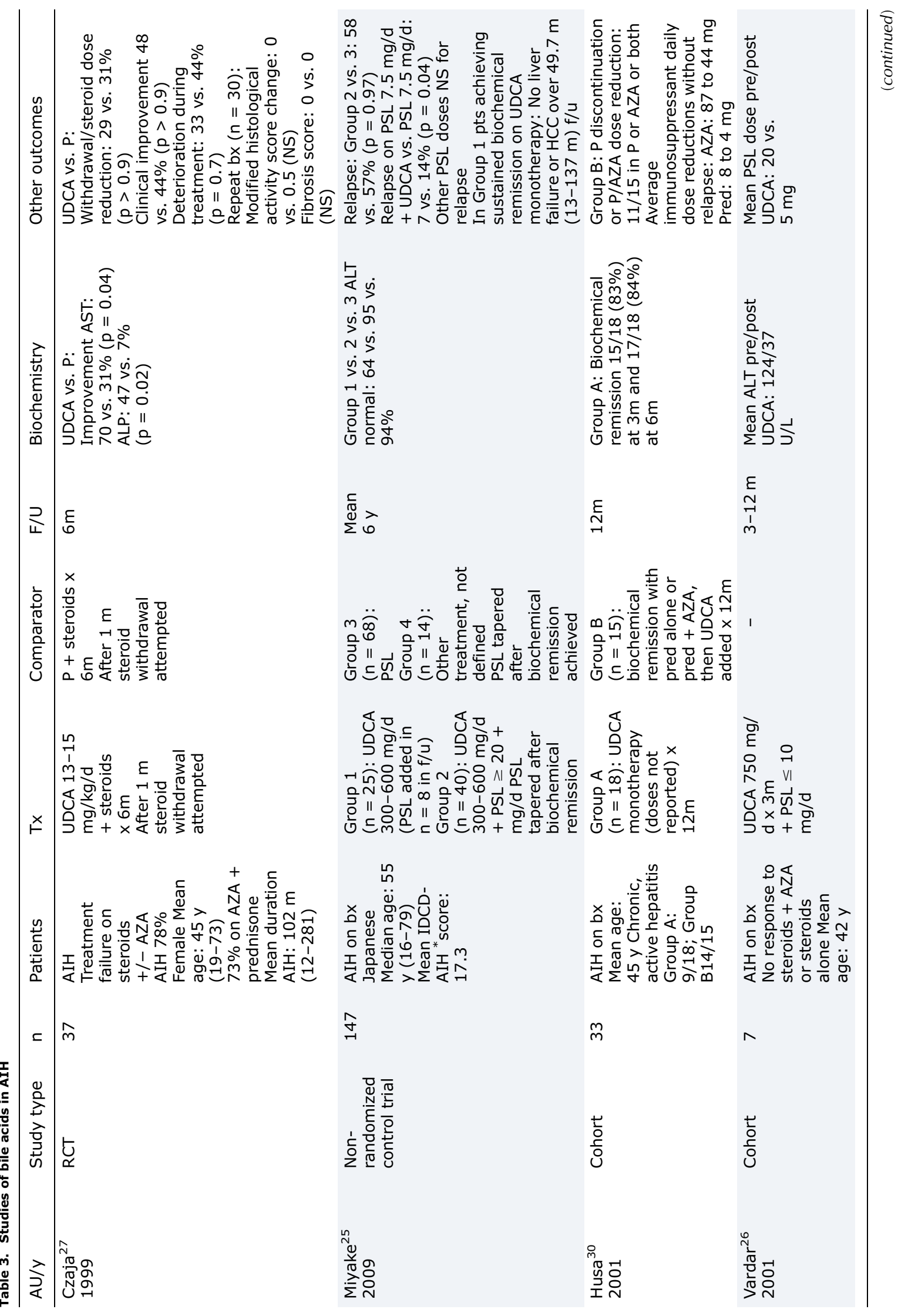




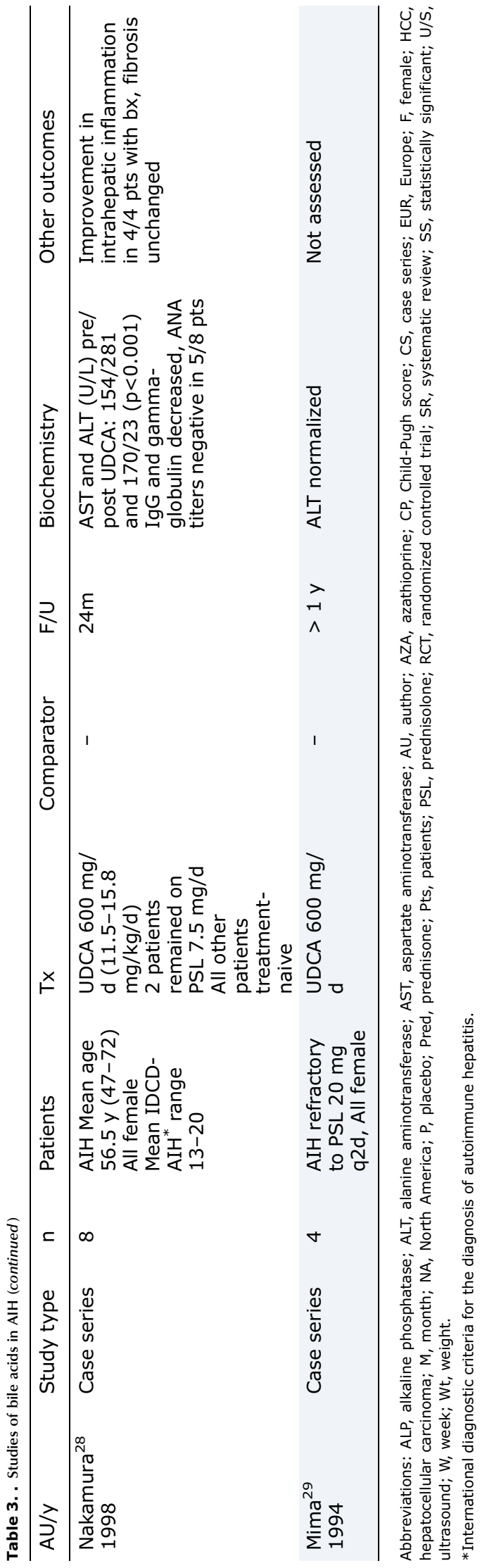

$A S T: A L T \geq 2$. Although non-specific, GGT is often elevated. ${ }^{19}$ The mechanisms of ALD are incompletely understood and pathology-dependent (steatosis vs. hepatitis). Evidence suggests that in patients with steatosis, alcohol stimulates lipogenesis and inhibits fatty acid oxidation, resulting in abnormal cellular signaling and maladaptive changes. Alcoholic hepatitis results from hepatocyte apoptosis incited by oxidative damage and stimulation of cytokine production. ${ }^{20}$ The mainstay of ALD treatment is abstinence from alcohol and nutritional support. Severe cases of hepatitis, however, may be managed with steroids or pentoxyifylline; although, evidence for benefit has been debated. ${ }^{21}$ Postulated benefits of UDCA in ALD are derived from limited human data demonstrating attenuation of lipid peroxidation, reduced cytokine activity and stabilization of cell membranes with improvement in fibrosis. ${ }^{22-24}$

In the majority of patients, UDCA did not affect clinical outcomes with only a marginal improvement in liver biochemistry (mainly GGT) shown in one pilot study. Lack of significant improvement in liver biochemistry in ALD patients, as compared to other patient populations reviewed, may be attributable to the severity of illness (most patients had significant cirrhosis with an average Child-Pugh score of BC) as well as persistent alcohol consumption. Given the limited data and lack of convincing benefits, UDCA does not appear to have a role in the management of ALD.

\section{AIH}

One RCT, 1 non-randomized controlled trial, 2 cohort trials and 2 case series examined the effect of UDCA in 236 patients with $\mathrm{AIH} .^{25-30}$ The majority of patients were females, aged $40-50$ years. UDCA doses ranged from $13-16 \mathrm{mg} / \mathrm{kg} / \mathrm{day}$ or were fixed at $600 \mathrm{mg} / \mathrm{day}$, with treatment durations of 3 months to $\geq 6$ years. Three trials enrolled patients with disease refractory to oral steroids \pm azathioprine. ${ }^{26,27,29}$

\section{Biochemistry}

Czaja et $\mathrm{al}^{27}$ randomized patients with $\mathrm{AIH}$ and suboptimal responses to steroids \pm azathioprine to receive add-on therapy with UDCA or placebo. Patients receiving UDCA had significant reductions in AST, ALT and alkaline phosphatase (ALP). Bilirubin, GGT, immunoglobulin (Ig) $G$ and albumin levels were not affected. A non-RCT concluded that UDCA monotherapy was not as effective as the combination with prednisolone for normalization of transaminases. Patients receiving UDCA monotherapy required longer treatment durations to achieve normalization. ${ }^{25}$ Similarly, observational studies concluded that addition of UDCA alone or in combination with steroids was associated with transaminase normalization. ${ }^{26,28-30}$

Two studies collected data on immunologic markers of $\mathrm{AIH}$. Nakamura et $\mathrm{al}^{28}$ reported decreased circulating IgG and gamma-globulin as well as achievement of negative antinuclear antibody (ANA) titers in 5/8 patients treated with UDCA over 2 years. Of note, patients were minimally symptomatic and considered to have mild disease not requiring steroids. Additionally, Husa et $\mathrm{al}^{30}$ reported significantly decreased concentrations of IgG, IgA and IgM at 6 months in patients receiving UDCA monotherapy. Bilirubin and circulating immune complexes remained unchanged. 


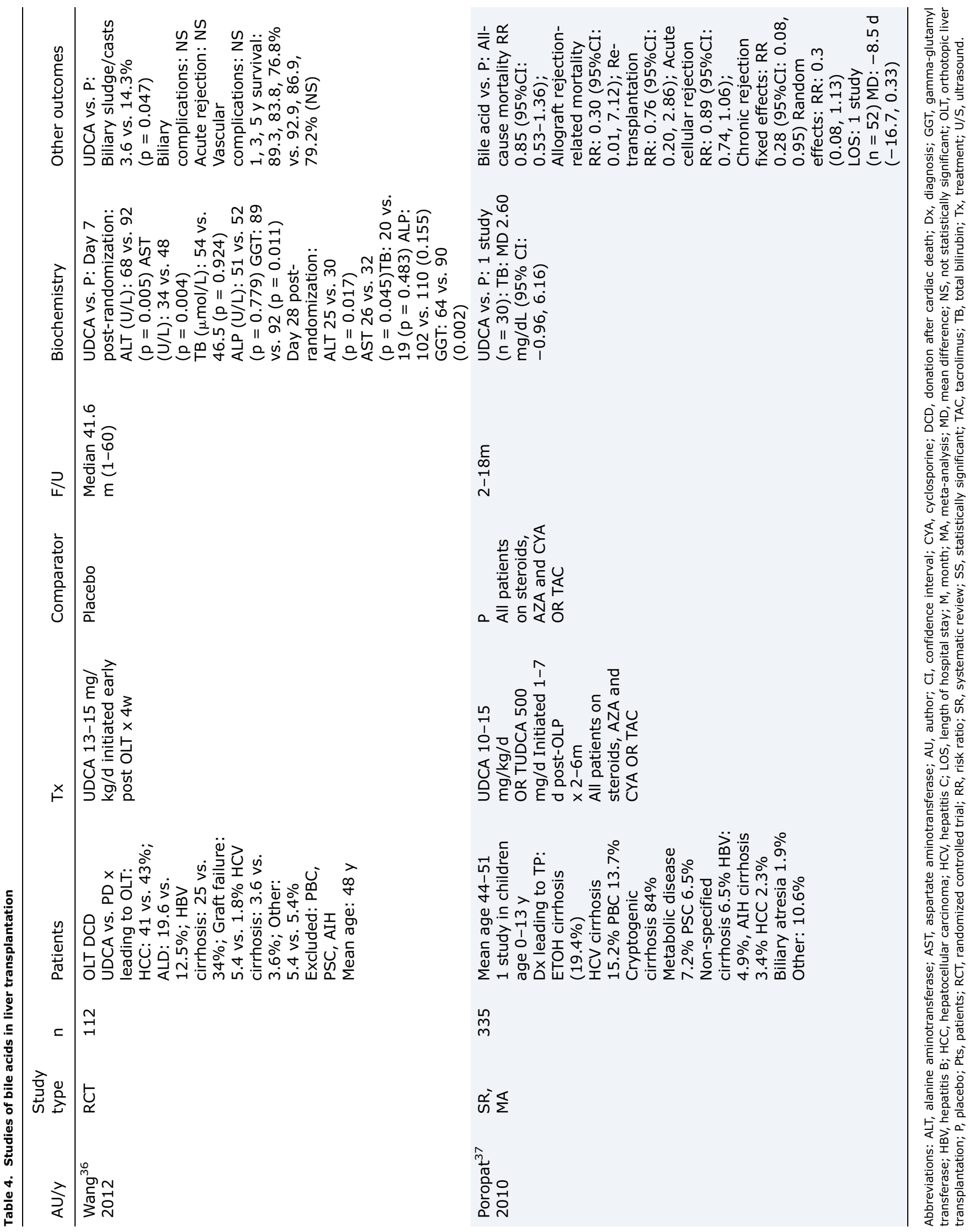




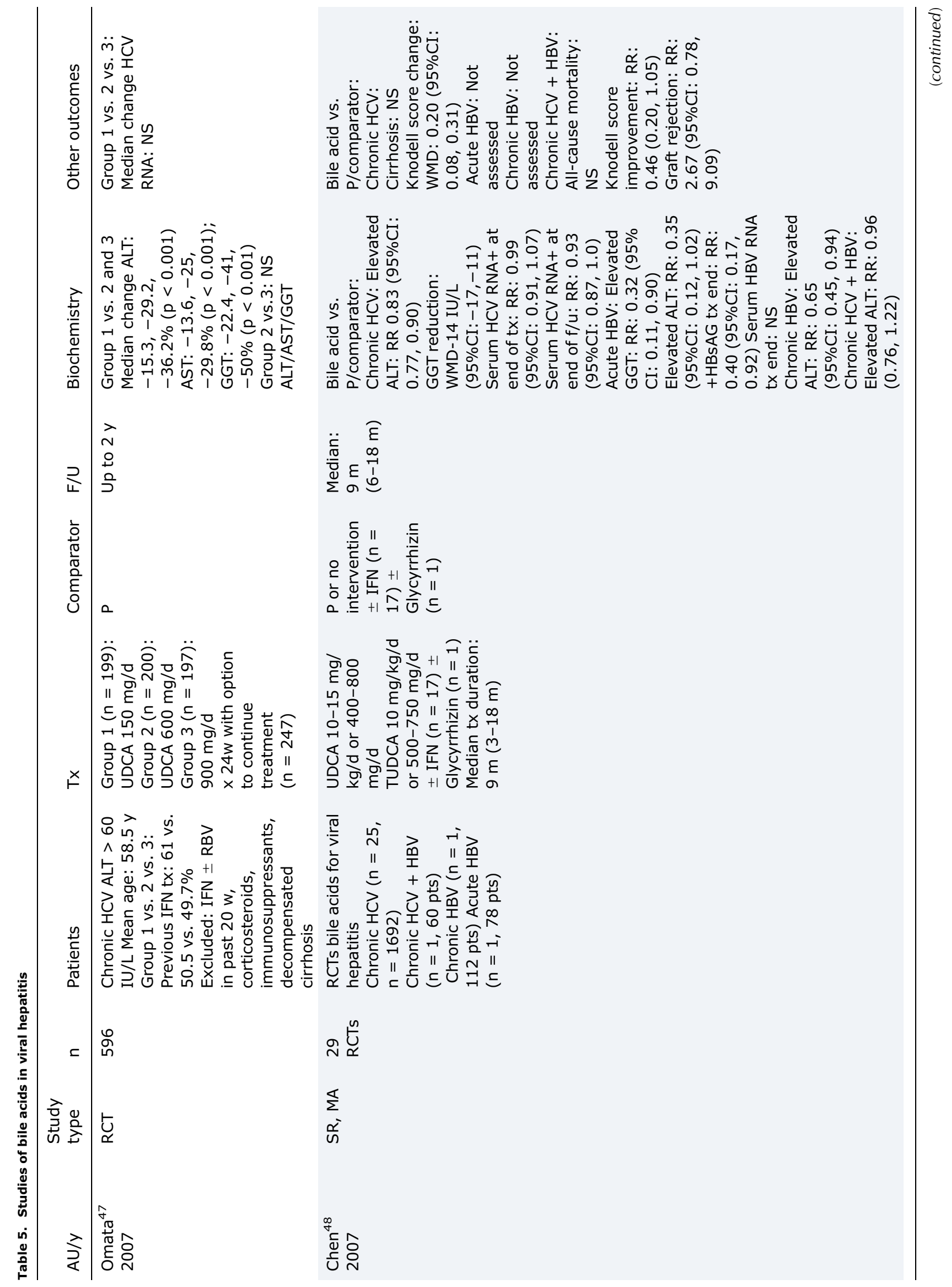




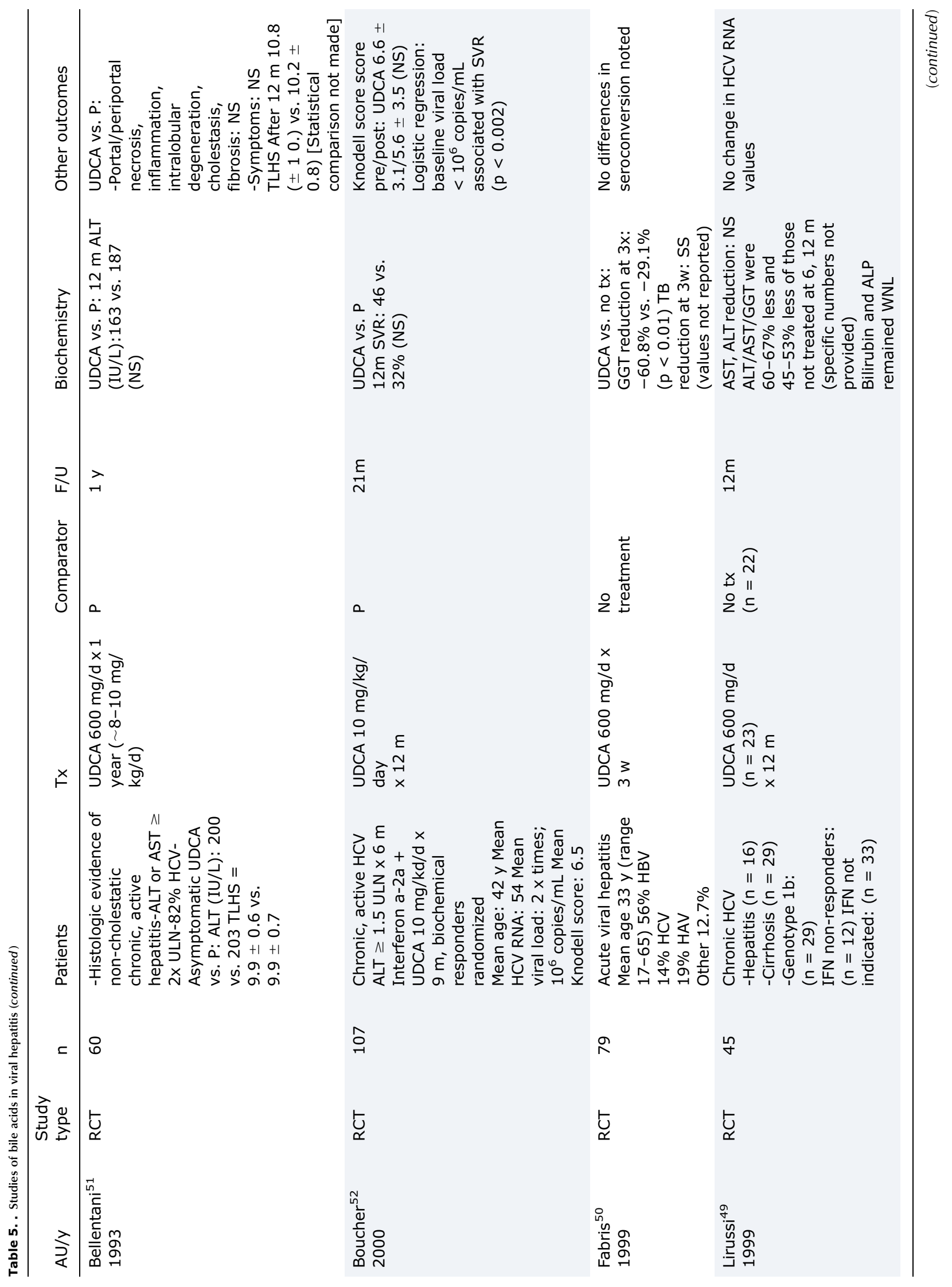




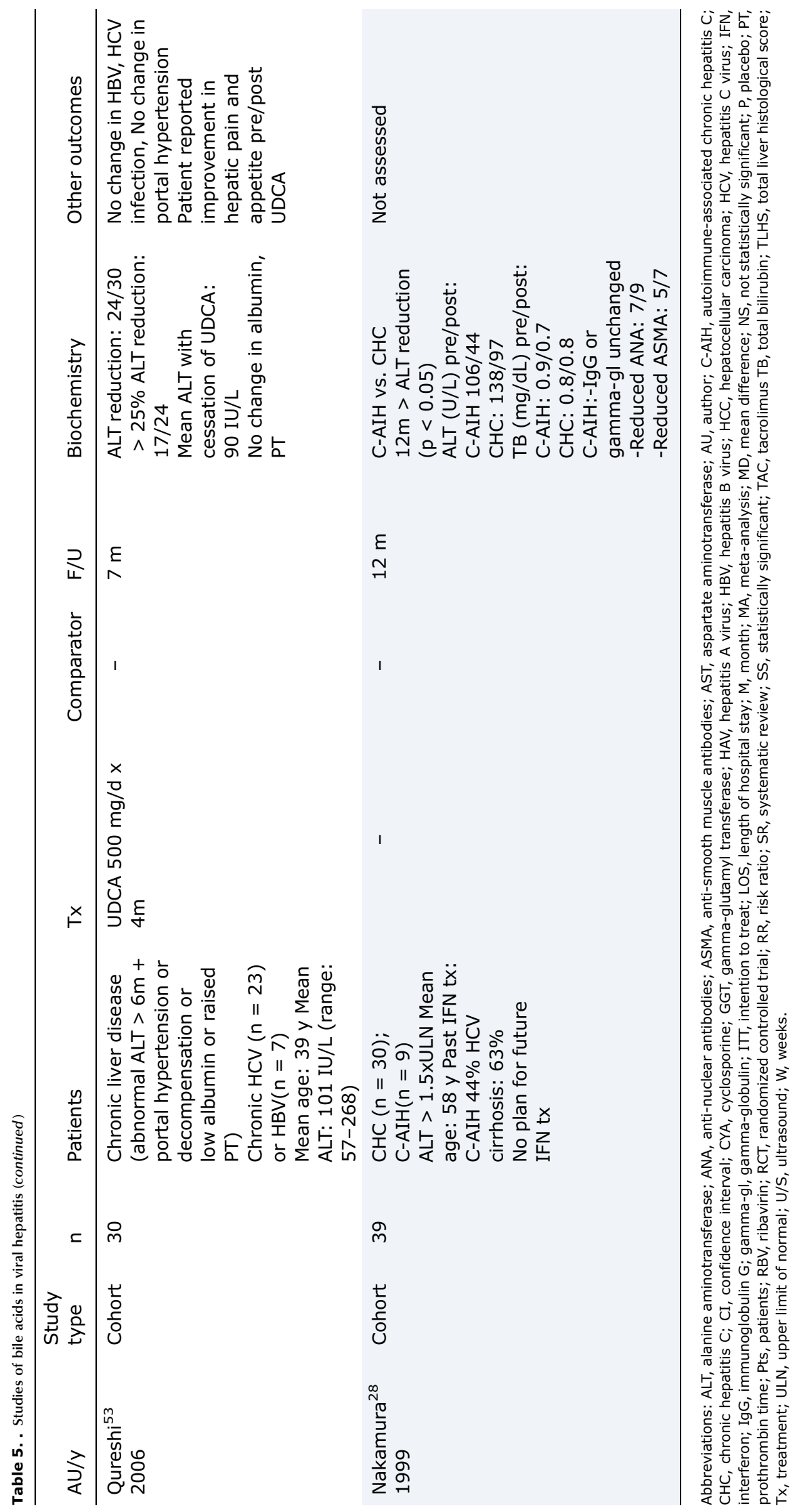


Histology

In studies performing repeat liver biopsies or ultrasound at post-UDCA initiation, no difference in disease progression was observed. ${ }^{25,27,28}$

Other clinical outcomes

Meta-analysis data from the largest number of patients in this review found no benefit for UDCA in facilitating steroid withdrawal. ${ }^{27}$ However, 4 individual studies not included in the meta-analysis reported ability to achieve lower steroid doses and greater successes in discontinuation when UDCA was used as adjunctive therapy. ${ }^{25,26,29,30}$

Summary

AIH is a chronic inflammatory condition of the liver, likely resulting from interplay of immune and environmental factors in genetically-vulnerable individuals. Presentation varies from asymptomatic to acute fulminant hepatic failure to end-stage cirrhosis. Diagnosis is guided by consideration of elevated transaminases, elevated gamma-globulin and/or IgG, presence of autoantibodies (ANA, smooth muscle antibody or anti-liver kidney microsome-1) and exclusion of other liver etiologies. Treatment is recommended when biochemical or histologic abnormalities exist and/or symptoms are present. ${ }^{31}$ Corticosteroids and azathioprine alone or in combination are mainstays of treatment. Although highly effective at inducing remission in $80-90 \%$ of patients, relapse after discontinuation of drug therapy is common. Considering the potential for significant long-term adverse effects with chronic use of these agents, an ideal adjunctive pharmacotherapy would allow for immunosuppressant minimization and prevention of relapse. Proposed UDCA mechanisms that may theoretically fulfill this role include immunomodulation through reduced hepatic expression of human leukocyte antigen (HLA) class 1 and attenuation of cytokine production leading to blunted immune system reactivity. ${ }^{32,33}$

Small sample sizes and heterogeneous patient populations limited generalizability of included studies of UDCA for AIH. Results were mixed in terms of liver biochemistry normalization, immunologic markers, steroid requirements and histologic improvement.

The magnitude of AST and gamma-globulin elevation has been associated with mortality in AIH patients. With this in mind, adjunctive UDCA in patients refractory to steroids and/ or azathioprine in an attempt to normalize these variables could be justified before attempting treatment with more toxic second-line options. Patients deriving the greatest benefits from UDCA were those who had less clinically severe disease. In practice, pharmacologic treatment of such patients may not be warranted, thus limiting the applicability of this data. Biochemical and histologic remission of AIH have been reported in case reports of patients on UDCA monotherapy. ${ }^{34,35}$ Considering the relatively benign side effect profile of UDCA, its use may be considered in patients with lower disease activity in an attempt to induce remission. In patients with more active AIH, UDCA may permit dosage reduction of immunosuppressants, particularly corticosteroids. Given the predilection of AIH for young females, this strategy could prove valuable in minimizing long-term side effects in this patient population. If used, a UDCA 13-15 $\mathrm{mg} / \mathrm{kg} /$ day in divided doses should be employed for a minimum of 3 months to assess benefit. Larger, randomized trials are required to fully elucidate the role of UDCA in AIH management.

\section{Liver transplantation}

One RCT and SR of 7 additional RCTs reported on UDCA or tauro-ursodeoxycholic acid (TUDCA) use post-transplantation for prevention of complications in the acute transplant period in 447 patients. UDCA/TUDCA doses ranged from 10-15 $\mathrm{mg} / \mathrm{kg} /$ day for $1-6$ months. In all trials, bile acids were initiated within the first week post-transplant. ${ }^{36,37}$ The majority of trials excluded patients with chronic cholestatic liver diseases, such as PBC or PSC.

\section{Biochemistry}

Only 1 trial included in the SR reported on liver biochemistry, citing no difference in bilirubin between patients treated with bile acids versus placebo. ${ }^{37}$ The additional RCT by Wang et $\mathrm{al}^{36}$ not included in the meta-analysis, found that administration of bile acids for the first 4 weeks post-liver transplant resulted in improvement in ALT, AST and GGT within 7 days, with no changes in bilirubin or ALP.

\section{Histology}

Poropat et $\mathrm{al}^{37}$ found a significant reduction in chronic rejection confirmed on biopsy for those receiving bile acids in a fixed effect model; however, this was not replicated in a random-effects model. Rates of acute rejection were not different for bile acids- versus placebo-treated patients. ${ }^{36,37}$

\section{Other clinical outcomes}

Poropat et $\mathrm{al}^{37}$ found no benefit for allograft rejection-related mortality or need for re-transplantation on meta-analysis. Neither trial found a difference in all-cause mortality up to 5 years post-transplant between recipients of UDCA versus placebo. ${ }^{36,37}$ Wang et $\mathrm{al}^{36}$ reported a $10.7 \%$ reduction in biliary sludge and casts in the year post-transplant with UDCA compared to placebo $(p=0.047)$.

\section{Summary}

Liver transplantation has become an increasingly common treatment of end-stage liver disease. Early post-operative complications may be surgical, medical or immunological in nature. Surgical complications commonly involve the biliary tract and may result in accumulation of toxic bile acids secondary to a biliary leaks or strictures. ${ }^{38}$ Administration of UDCA to alter the proportion of hydrophobic to hydrophilic bile acids may exert cytoprotective effects in these patients. Immunologic complications are related to rejection, with concern of acute cellular rejection (ACR) in the early postoperative period. Early ACR typically occurs within the first few weeks after transplantation and is characterized by abnormal liver biochemistry and inflammatory histologic changes. Episodes usually result in no long-term impact on graft survival and are managed with pulse steroids and/or increased immunosuppression. An exception is HCV patients, in whom ACR treatment has been associated with increased risk of cirrhosis and mortality. ${ }^{38,39}$ 
In liver transplant patients, UDCA may theoretically prevent allograft rejection by alteration of major histocompatibility complex (MHC) class I antigen expression in bile duct epithelium and central vein endothelium. ${ }^{40,41}$ Despite this, no differences in acute graft rejection were observed in the reviewed studies. Additionally, recognition of MHC class II antigens by CD4 $\mathrm{T}$ cells has been identified as an inciting mechanism in acute cellular rejection. ${ }^{42}$ As UDCA is thought only to impact $\mathrm{MCH}$ class I antigens, there may be no role for mitigation of rejection episodes by this pathway. Theoretically, as rejection risk is highest early in the post-transplant period, initiation of UDCA pre-operatively may be required to realize any benefit. The ability of bile acids to act as immunosuppressant-sparing agents was observed in some studies, but further evaluation of this outcome is needed. Although some trials found benefit for reduced chronic rejection and transplant-related hospitalizations with UDCA compared to controls, these results must be interpreted with caution as sample sizes were small and the trials were considered high-risk for bias. Bile acid administration immediately posttransplant may improve liver biochemistry, but the differences observed were minimal and of questionable clinical relevance. There is currently no strong evidence to support or refute bile acids for management of liver-transplanted patients with non-cholestatic liver disease.

Another complication that may occur during transplantation is ischemic-reperfusion injury (IRI). Although underlying molecular mechanisms have yet to be elucidated, IRI induces graft dysfunction via direct cellular toxicity occurring during restoration of allograft blood flow intraoperatively. ${ }^{43}$ Although anecdotally UDCA may be used peri-transplant in an attempt to attenuate ischemic damage, no human data was found to support this practice. One animal model found no change in biochemical, hemodynamic or histologic parameters with UDCA administration post-transplant. ${ }^{44}$ Conversely, a rat study showed that intravenous infusion of UDCA at the time of graft reperfusion led to reduced release of liver enzymes and mitigated toxic effects of endogenous bile salts by improving graft bile flow. ${ }^{45}$ An additional animal study showed that administration of enteral UDCA to the liver donor at 3 hours pre-transplant led to lower ALT and less hepatocyte apoptosis post-transplant. ${ }^{46}$

There is currently no strong evidence to support or refute bile acids for management of liver-transplanted patients with non-cholestatic liver disease pre- or post-transplant.

\section{Viral hepatitis}

An SR of 29 RCTs, 5 RCTs not included in the SR, 2 cohort studies and 1 case report evaluated UDCA or TUDCA for patients with viral hepatitis. The majority of included patients had HCV disease and had previously failed or were not candidates for interferon. UDCA/TUDCA doses ranged from $150-900 \mathrm{mg} /$ day with treatment durations from 3 weeks to 2 years. ${ }^{47-55}$

\section{Biochemistry}

A Cochrane systematic review by Chen et al ${ }^{48}$ of 29 RCTs comparing any dose or duration of bile acids with placebo or no intervention for treatment of patients with HBV or HCV found significant decreases in serum transaminases with acute HBV and chronic HBV and HCV. One included trial found UDCA reduced risk of hepatitis $B$ surface antigen positivity and HBV DNA levels, as compared to placebo in patients with acute HBV. Viral loads were not affected by bile acid use in the other included studies. RCTs not included in the Cochrane review and observational studies were congruent with these findings, demonstrating persistence of viral loads in the setting of improving liver biochemistry with bile acid use. ${ }^{47,49-54}$ One dose-finding study reported superiority of UDCA at $600 \mathrm{mg} /$ day over the dose of $150 \mathrm{mg} /$ day for ALT, AST and GGT improvement. Doses of $900 \mathrm{mg} /$ day provided no additional benefit. ${ }^{47}$

\section{Histology}

In their meta-analysis, Chen et $\mathrm{al}^{48}$ report a significant, albeit small, increase in Knodell scores in patients on bile acids compared to controls. Other trials reviewed did not find any significant changes in liver fibrosis scores. $48,51-53$

\section{Other clinical outcomes}

A case report by Anzi et al $^{55}$ describes a 42 -year-old woman with chronic HCV with lack of response to interferon. Implementation of combined low-dose interferon and UDCA led to successful progression disease-free survival in up to 4 years of follow-up. An observational trial reported subjective improvement in abdominal pain and appetite after initiation of UDCA. ${ }^{53}$

\section{Summary}

The primary mechanism of purported benefit of exogenous bile acids in management of hepatitis involves anti-apoptotic mechanisms. All patients included in the studies had transaminase elevation. There is some data to suggest that improvement of elevated transaminases, as was seen in most studies, may mitigate disease progression in HCV. ${ }^{56}$ Despite enzyme improvement, viral loads were not significantly impacted by bile acid use. Interestingly, Nakamura et $\mathrm{al}^{57}$ noted a greater benefit of UDCA in patients with HCV and autoimmune features (elevated IgG, positive ANA or antismooth muscle antibodies (ASMA)) lending support to the hypothesis that an immunomodulatory effect of UDCA may be responsible for any observed benefits. The bulk of data evaluating UDCA for viral hepatitis was in chronic HCV patients with past or concurrent interferon use. Recent availability of direct acting antiviral agents has revolutionized HCV treatment, producing sustained virologic responses of $>90 \%$ for certain HCV genotypes. Superior efficacy to interferon and excellent tolerability have positioned these agents as first line HCV treatment options, arguably rendering pursuit of adjunctive therapies for HCV unnecessary. Finally, as the natural progression of viral hepatitis-induced cirrhosis is slow, the duration of these studies precludes meaningful interpretation of histologic outcomes and assessment of risk for HCC and liver-related mortality. The currently available evidence does not support use of bile acids in treatment of acute or chronic HBV or HCV.

\section{Safety}

In the prospective trials reviewed, adverse effects with bile acids were limited to minor gastrointestinal complaints (most commonly diarrhea) and did not occur at increased frequency compared to controls. If used, UDCA should be administered 
in divided doses to minimize gastrointestinal distress. Although not reported in the reviewed studies, PBC literature has exhibited risk of weight gain with UDCA at the doses of $13-15 \mathrm{mg} / \mathrm{kg} / \mathrm{day}$, plateauing at 5 pounds during the first year of use. ${ }^{58}$ Lastly, as administration of bile acids was often limited to 12 months durations or less, long-term side effects for non-cholestatic indications cannot be excluded. In clinical practice, UDCA is typically dosed empirically at $450-600 \mathrm{mg} /$ day, administered in divided doses. For an average $70 \mathrm{~kg}$ male, this would be lower than the $13-15 \mathrm{mg} / \mathrm{kg}$ employed in many of the included studies.

\section{Limitations}

This review has several limitations. It included only articles published in English; however, all abstracts from identified articles (English and non-English) were screened and no abstracts of non-English articles appeared to contain relevant content. Studies assessed were of varying methodological quality and of small sample size. The majority of studies evaluated surrogate markers of liver disease and were not adequately powered to assess clinically relevant long-term outcomes. Although we intended to review evidence for UDCA, a minority of studies assessed patients treated with its taurine conjugate, TUDCA. TUDCA has demonstrated comparable efficacy and safety to UDCA, and therefore this should not have affected outcomes. ${ }^{59}$ Most included studies were published $\geq 10$ years ago; however, with the exception of viral hepatitis treatments, the management of non-cholestatic liver disease has not changed so dramatically as to impact the relevance and applicability of these results.

A systematic literature review on use of exogenous, hydrophilic bile acids for treatment of non-cholestatic liver disease revealed heterogeneous data comprised of variable patient populations and methodologies, thus limiting generalizability. Bile acid use may be associated with improved normalization of liver biochemistry in NAFLD, AIH, HBV and $\mathrm{HCV}$ patients, but these findings have limited clinical relevance. Normalization of liver biochemistry did not correlate to improvement in histologic disease in the majority of studies. Larger studies would be required for proper evaluation of the impact of bile acid administration on clinically meaningful outcomes, such as disease burden and including progression to cirrhosis and HCC.

\section{Conflict of interest}

None

\section{Author contributions}

Formulated the research question and participated in development of the search strategy (JR, TH, NP, EMY), performed the literature search and drafted the initial manuscript (JR), revised the manuscript for important intellectual content $(T H$, MA, VMV, SRE, NP, EMY).

\section{REFERENCES}

[1] Poupon R. Ursodeoxycholic acid and bile-acid mimetics as therapeutic agents for cholestatic liver diseases: an overview of their mechanisms of action. Clin Res Hepatol Gastroenterol 2012;36:S3-S12. doi: 10.1016/S2210-7401(12) 70015-3.
[2] Chiang JY. Bile acids: regulation of synthesis. J Lipid Res 2009;50:19551966. doi: 10.1194/jlr.R900010-JLR200.

[3] Trauner M, Graziadei IW. Review article: mechanisms of action and therapeutic applications of ursodeoxycholic acid in chronic liver diseases. Aliment Pharmacol Ther 1999;13:979-996. doi: 10.1046/j.1365-2036.1999.00596.x.

[4] Lindor KD, Gershwin ME, Poupon R, Kaplan M, Bergasa NV, Heathcote EJ. Primary biliary cirrhosis. Hepatology 2009;50:291-308. doi: 10.1002/hep 22906.

[5] Chapman R, Fevery J, Kalloo A, Nagorney DM, Boberg KM, Shneider B, et al. Diagnosis and management of primary sclerosing cholangitis. Hepatology 2010;51:660-678. doi: 10.1002/hep.23294.

[6] Beuers U, Boyer JL, Paumgartner G. Ursodeoxycholic acid in cholestasis: potential mechanisms of action and therapeutic applications. Hepatology 1998;28:1449-1453. doi: 10.1002/hep.510280601.

[7] Xiang Z, Chen YP, Ma KF, Ye YF, Zheng L, Yang YD, et al. The role of ursodeoxycholic acid in non-alcoholic steatohepatitis: a systematic review. BMC Gastroenterol 2013;13:140. doi: 10.1186/1471-230X-13-140.

[8] Pietu F, Guillaud O, Walter T, Vallin M, Hervieu V, Scoazec JY, et al. Ursodeoxycholic acid with vitamin $\mathrm{E}$ in patients with nonalcoholic steatohepatitis: long-term results. Clin Res Hepatol Gastroenterol 2012;36:146-155. doi: 10.1016/j.clinre.2011.10.011.

[9] Ersöz G, Günşar F, Karasu Z, Akay S, Batur Y, Akarca US. Management of fatty liver disease with vitamin $\mathrm{E}$ and $\mathrm{C}$ compared to ursodeoxycholic acid treatment. Turk J Gastroenterol 2005;16:124-128.

[10] Madan K, Batra Y, Gupta DS, Chander B, Anand Rajan KD, Singh R, et al. Vitamin E-based therapy is effective in ameliorating transaminasemia in nonalcoholic fatty liver disease. Indian J Gastroenterol 2005;24:251-255.

[11] Troisi G, Crisciotti F, Gianturco V, D'Ottavio E, Lo Iacono C, Formosa V, et al. The treatment with ursodeoxycholic acid in elderly patients affected by NAFLD and metabolic syndrome: a case-control study. Clin Ter 2013;164: 203-207.

[12] Chalasani N, Younossi Z, Lavine JE, Diehl AM, Brunt EM, Cusi K, et al. The diagnosis and management of non-alcoholic fatty liver disease: Practice guideline by the American Gastroenterological Association, American Association for the Study of Liver Diseases, and American College of Gastroenterology. Gastroenterology 2012;142:1592-1609. doi: 10.1053/j.gastro. 2012.04.001.

[13] Pathil A, Mueller J, Warth A, Chamulitrat W, Stremmel W. Ursodeoxycholyl Iysophosphatidylethanolamide improves steatosis and inflammation in murine models of nonalcoholic fatty liver disease. Hepatology 2012;55: 1369-1378. doi: 10.1002/hep.25531.

[14] Neuman $M$, Angulo $P$, Malkiewicz I, Jorgensen $R$, Shear $N$, Dickson ER, et al. Tumor necrosis factor-alpha and transforming growth factor-beta reflect severity of liver damage in primary biliary cirrhosis. J Gastroenterol Hepato 2002;17:196-202. doi: 10.1046/j.1440-1746.2002.02672.x.

[15] Rodrigues CM, Fan G, Ma X, Kren BT, Steer CJ. A novel role for ursodeoxycholic acid in inhibiting apoptosis by modulating mitochondrial membrane perturbation. J Clin Invest 1998;101:2790-2799. doi: 10.1172/JCI1325.

[16] Mofrad P, Contos MJ, Haque M, Sargeant C, Fisher RA, Luketic VA, et al. Clinical and histologic spectrum of nonalcoholic fatty liver disease associated with normal ALT values. Hepatology 2003;37:1286-1292. doi: 10 . 1053/jhep.2003.50229.

[17] Pelletier G, Roulot D, Davion T, Masliah C, Causse X, Oberti F, et al. A randomized controlled trial of ursodeoxycholic acid in patients with alcohol-induced cirrhosis and jaundice. Hepatology 2003;37:887-892. doi: 10.1053/jhep. 2003.50118.

[18] Plevris JN, Hayes PC, Bouchier IAD. Ursodeoxycholic acid in the treatment of alcoholic liver-disease. Eur J Gastroen Hepat 1991;3:653-656.

[19] O'Shea RS, Dasarathy S, McCullough AJ. Alcoholic liver disease. Hepatology 2010;51:307-328. doi: 10.1002/hep.23258.

[20] Gao B, Bataller R. Alcoholic liver disease: pathogenesis and new therapeutics targets. Gastroenterology 2011;141:1572-1585. doi: 10.1053/j.gastro. 2011. 09.002 .

[21] Thursz MR, Richardson P, Allison M, Austin A, Bowers M, Day CP, et al. Prednisolone or pentoxifylline for alcoholic hepatitis. N Engl J Med 2015;372: 1619-1628. doi: 10.1056/NEJMoa1412278.

[22] Preobrazhenskiĭ VN. Taianovskiĭ VIu. Efficacy of ursodeoxycholic acid (UDCA) in the treatment of chronic alcoholic hepatitis. Klin Med (Mosk) 1998;76: 26-28.

[23] Belonovskaia EB, Naruta EE, Lukivskaia OIa, Abakumov VZ, Buko VU. Preventive administration of new UDCA derivatives in experimental alcoholic steatohepatitis. Eksp Klin Farmakol 2013;76:25-29.

[24] Virstyuk N, Deltsova O, Geraschenko S, Kovalchuk L. Effects of ursodeoxycholic acid and L-ornithinel-aspartate on hepatocytes changes in ALD patients. Journal of Hepatology. Conference: 48th Annual Meeting of the European Association for the Study of the Liver, International Liver Congress 2013 Amsterdam Netherlands. Conference Publication: (S226), 2013.

[25] Miyake Y, Iwasaki Y, Kobashi H, Yasunaka T, Ikeda F, Takaki A, et al. Efficacy of ursodeoxycholic acid for Japanese patients with autoimmune hepatitis. Hepatol Int 2009;3:556-562. doi: 10.1007/s12072-009-9155-9. 
[26] Vardar R, Ersöz G, Karasu Z, Akarca US. Ursodeoxycholic acid can be used as a steroid-tapering drug in the treatment of autoimmune hepatitis. Turk J Gastroenterol 2001;12:249-252.

[27] Czaja AJ, Carpenter HA, Lindor KD. Ursodeoxycholic acid as adjunctive therapy for problematic type 1 autoimmune hepatitis: a randomized placebocontrolled treatment trial. Hepatology 1999;30:1381-1386. doi: 10.1002/ hep. 510300603.

[28] Nakamura K, Yoneda M, Yokohama S, Tamori K, Sato Y, Aso K, et al. Efficacy of ursodeoxycholic acid in Japanese patients with type 1 autoimmune hepatitis. J Gastroenterol Hepatol 1998;13:490-495. doi: 10.1111/j.14401746.1998.tb00674.x.

[29] Mima S, Sekiya C, Kanagawa H, Uchida T. Ursodeoxycholic acid (UDCA) therapy for autoimmune hepatitis. Int Hepatol Commun 1994;2:207-212. doi: $10.1016 / 0928-4346(94) 90072-8$.

[30] Husa P, Chalupa P, Husová L. Ursodeoxycholic acid is efficacious also in the treatment of autoimmune hepatitis. Gastroenterol Pol 2001;8:149-152.

[31] Manns MP, Czaja AJ, Gorham JD, Krawitt EL, Mieli-Vergani G, Vergani D, et al. Diagnosis and management of autoimmune hepatitis. Hepatology 2010;51: 2193-2213. doi: 10.1002/hep.23584.

[32] Yoshikawa M, Tsujii T, Matsumura K, Yamao J, Matsumura Y, Kubo R, et al. Immunomodulatory effects of ursodeoxycholic acid on immune responses. Hepatology 1992;16:358-364. doi: 10.1002/hep.1840160213.

[33] Hirano F, Tanaka H, Makino Y, Okamoto K, Makino I. Effects of ursodeoxycholic acid and chenodeoxycholic acid on major histocompatibility complex class I gene expression. J Gastroenterol 1996;31:55-60. doi: 10.1007/ BF01211187.

[34] Duclos-Vallée JC, Di Martino V, Cazier A, Ballot E, Johanet C, Yamamoto AM, et al. Remission with ursodeoxycholic acid of type 1 autoimmune hepatitis resistant to azathioprine and steroids. Gastroenterol Clin Biol 2005;29: 1173-1176. doi: 10.1016/S0399-8320(05)82185-2.

[35] Matsushita M, Takemura T, Imai Y, Takimoto K, Kuchide M, Kajikawa H, et al. A case of probable autoimmune hepatitis from which the persistence of hepatitis A IgM antibody, and the improvement was pathologically obtained by ursodeoxycholic acid medication. Nihon Shokakibyo Gakkai Zasshi 2008; 105:1367-1374.

[36] Wang SY, Tang HM, Chen GQ, Xu JM, Zhong L, Wang ZW, et al. Effect of ursodeoxycholic acid administration after liver transplantation on serum liver tests and biliary complications: a randomized clinical trial. Digestion 2012;86:208-217. doi: 10.1159/000339711

[37] Poropat G, Giljaca V, Stimac D, Gluud C. Bile acids for liver-transplanted patients. Cochrane Database Syst Rev 2010;17:CD005442. doi: 10. 1002/14651858.cd005442.pub2.

[38] Mueller AR, Platz KP, Kremer B. Early postoperative complications following liver transplantation. Best Pract Res Clin Gastroenterol 2004;18:881-900. doi: 10.1016/S1521-6918(04)00109-X.

[39] Thurairajah PH, Carbone M, Bridgestock H, Thomas P, Hebbar S, Gunson BK, et al. Late acute liver allograft rejection; a study of its natural history and graft survival in the current era. Transplantation 2013;95:955-959. doi: 10. 1097/TP.0b013e3182845f6c.

[40] Calmus Y, Gane P, Rouger P, Poupon R. Hepatic expression of class I and class II major histocompatibility complex molecules in primary biliary cirrhosis: effect of ursodeoxycholic acid. Hepatology 1990;11:12-15. doi: 10.1002/ hep. 1840110104.

[41] Perez MJ, Briz O. Bile-acid-induced cell injury and protection. World J Gastroenterol 2009;15:1677-1689. doi: 10.3748/wjg.15.1677.

[42] Vierling JM. Immunologic mechanisms of hepatic allograft rejection. Semin Liver Dis 1992;12:16-27. doi: 10.1055/s-2007-1007373.
[43] Peralta $C$, Jiménez-Castro MB, Gracia-Sancho J. Hepatic ischemia and reperfusion injury: effects on the liver sinusoidal milieu. J Hepatol 2013;59:10941106. doi: 10.1016/j.jhep.2013.06.017.

[44] Chazouillères O, Ballet F, Legendre C, Bonnefis MT, Rey C, Chrétien Y, et al. Effect of bile acids on ischemia-reperfusion liver injury. J Hepatol 1991;13: 318-322. doi: 10.1016/0168-8278(91)90075-M.

[45] Hertl M, Clausnitzer N, Rogiers X, Jung R, Broelsch CE. Infusion of hydrophilic bile salts protects the liver from reperfusion injury in the rat. Transplant Proc 1997;29:386-387. doi: 10.1016/S0041-1345(96)00130-3.

[46] Nowak G, Norén UG, Wernerson A, Marschall HU, Möller L, Ericzon BG. Enteral donor pre-treatment with ursodeoxycholic acid protects the liver against ischaemia-reperfusion injury in rats. Transpl Int 2005;17:804-809. doi: 10.1111/j.1432-2277.2004.tb00514.x.

[47] Omata M, Yoshida H, Toyota J, Tomita E, Nishiguchi S, Hayashi N, et al. A large-scale, multicentre, double-blind trial of ursodeoxycholic acid in patients with chronic hepatitis C. Gut 2007;56:1747-1753. doi: 10.1136/ gut.2007.120956.

[48] Chen W, Liu J, Gluud C. Bile acids for viral hepatitis. Cochrane Database Syst Rev 2007;17:CD003181. doi: 10.1002/14651858.cd003181.pub2.

[49] Lirussi F, Beccarello A, Bortolato L, Morselli-Labate AM, Crovatto M, Ceselli S, et al. Long-term treatment of chronic hepatitis $C$ with ursodeoxycholic acid: influence of HCV genotypes and severity of liver disease. Liver 1999;19: 381-388. doi: 10.1111/j.1478-3231.1999.tb00066.x.

[50] Fabris P, Tositti G, Mazzella G, Zanetti AR, Nicolin R, Pellizzer G, et al. Effect of ursodeoxycholic acid administration in patients with acute viral hepatitis: a pilot study. Aliment Pharmacol Ther 1999;13:1187-1193. doi: 10.1046/j. 1365-2036.1999.00592.x.

[51] Bellentani S, Podda M, Tiribelli C, Callea F, Marazzi M, Sodde M, et al. Ursodiol in the long-term treatment of chronic hepatitis: a double-blind multicenter clinical trial. J Hepatol 1993;19:459-464. doi: 10.1016/S0168-8278(05) 80558-6.

[52] Boucher E, Guyader D, Jacquelinet S, Andre P, Mendler MH, Turlin B, et al. Interferon and ursodeoxycholic acid combined therapy in chronic viral $C$ hepatitis: controlled randomized trial in 203 patients. Dig Liver Dis 2000; 32:29-33. doi: 10.1016/S1590-8658(00)80041-9.

[53] Qureshi H, Mehdi I, Ahmed WU, Alam SE. Role of ursodeoxycholic acid in lowering ALT in chronic liver disease. J Pak Med Assoc 2006;56:130-131.

[54] Nakamura K, Yoneda M, Takamoto S, Nakade Y, Yokohama S, Tamori K, et al. Effect of ursodeoxycholic acid on autoimmune-associated chronic. J Gastroenterol Hepatol 1999;14:413-418. doi: 10.1046/j.1440-1746.1999.01898.x.

[55] Anzai T, Miyake $Y$, Yasuhara H, Miyatake H, Asagi A, Moriya A, et al. Long-term combined therapy with very-low-dose peginterferon and ursodeoxycholic acid decreased the spleen size in a patient with hepatitis $C$ virus-related cirrhosis. Intern Med 2013;52:363-367. doi: 10.2169/internalmedicine.52.8939.

[56] Mathurin P, Moussalli J, Cadranel JF, Thibault V, Charlotte F, Dumouchel P, et al. Slow progression rate of fibrosis in hepatitis $C$ virus patients with persistently normal alanine transaminase activity. Hepatology 1998;27:868-872. doi: 10.1002/hep.510270333.

[57] Elbaz T, El-Kassas M, Esmat G. New era for management of chronic hepatitis $C$ virus using direct antiviral agents: A review. J Adv Res 2015;6:301-310. doi: 10.1016/j.jare.2014.11.004.

[58] Siegel JL, Jorgensen R, Angulo P, Lindor KD. Treatment with ursodeoxycholic acid is associated with weight gain in patients with primary biliary cirrhosis. J Clin Gastroenterol 2003;37:183-185. doi: 10.1097/00004836-20030800000018.

[59] Larghi A, Crosignani A, Battezzati PM, De Valle G, Allocca M, Invernizzi P, et al. Ursodeoxycholic and tauro-ursodeoxycholic acids for the treatment of primary biliary cirrhosis: a pilot crossover study. Aliment Pharmacol Ther 1997;11:409-414. doi: 10.1046/j.1365-2036.1997.124295000.x. 